\title{
The Effects of Pre-Surgical Education on Patient Expectations in Total Knee Arthroplasties
}

\author{
Steven Furney ${ }^{1}$, Natasha Montez ${ }^{2}$ \\ ${ }^{1}$ Department of Health and Human Performance, Texas State University, San Marcos, USA \\ ${ }^{2}$ Invictus Health Solutions, New Braunfels, USA \\ Email: sf02@txstate.edu, tashamontezphd@gmail.com
}

Received 3 October 2015; accepted 1 December 2015; published 4 December 2015

Copyright (C) 2015 by authors and Scientific Research Publishing Inc.

This work is licensed under the Creative Commons Attribution International License (CC BY). http://creativecommons.org/licenses/by/4.0/

(c) (i) Open Access

\begin{abstract}
As patients prepare for total-knee arthroplasty surgery, they have many expectations related to their long-term recovery and function. This research examined whether the use of a pre-surgical patient education class with an additional long-term expectation module addressing recovery during the first 12 months after surgery was more effective in modifying participant's pre-surgical expectations than participants receiving the standard pre-surgical education class alone. Prior to the class each participant completed one disease-specific instrument, a general-health survey, and a total-knee replacement expectation survey. After the class, each participant once again completed the total-knee replacement survey. Included in the study were 42 participants who were enrolled in a pre-surgical education course that was randomized. The participants in the control group received the standard pre-surgical education addressing pre-surgical topics. The participants in the intervention group received the standard pre-surgical education plus an additional module that specifically addressed long-term recovery and function up to 12 months post surgery. The primary outcome of the data revealed that participants' who received the standard pre-surgical education with the additional module and who had an educational level higher than highschool, had expectations that were able to be modified to coincide with the surgeons' expectations.
\end{abstract}

\section{Keywords}

Osteoarthritis, Total-Knee Arthroplasty, Pre-Surgical Education, Patient Expectations

\section{Introduction}

Each year over 27 million Americans are diagnosed with osteoarthritis (OA) [1]. OA is characterized by the ero- 
sion of articular cartilage, osteophyte formation, and joint-space narrowing. Specific to the knee, OA is reported to affect approximately $10 \%$ of the United States population over the age of fifty-five [2]. It is the leading cause of chronic disability in those older than 70 years, costing the US greater than 100 billion dollars annually [3]. Frequently affected sites include hands, knees, hips, and spine. Pathological features include inflammation, pain, stiffness, joint deformity, and loss of mobility [4]. Some high risk factors of OA include aging, genetics, traumatic injury, gender, obesity, repetitive use, and abnormal loading [5]. Arthritis for millions can be described as excruciatingly painful, debilitating, and at its most severe stage, incapacitating. Treatment options range from conservative (i.e. anti-inflammation medications, bracing, and visco supplement injections) to surgical interventions (i.e. total-knee arthroplasty/total-knee replacement). Total-knee arthroplasties (TKA) or total-knee replacements, have been shown to be a cost-effective, successful, reliable, and a low-risk treatment option for those suffering from moderate to severe end-stage OA [2] [6] [7]. By the year 2030, the demand for primary totalknee arthroplasties is projected to increase by $673 \%$, thus translating into 3.48 million total-knee replacements to be performed annually [1]. The ultimate goal of primary TKA is to reduce knee-joint pain, improve range of motion, improve functional status of the individual, and to allow improvement of the patient's overall quality of life [6]. In the offices of orthopedic surgeons, the number of patients deemed appropriate candidates for TKA continues to increase. Patients are seen daily who suffer and live with the pain of moderate to severe OA. When a patient is deemed an appropriate candidate for a TKA, a number of emotions develop along with concerns and various expectations. While some patients will have realistic expectations of surgical outcomes, many will have expectations that exceed the functional goals of the surgery.

In the field of orthopedic medicine, the need for patient education to address expectations of TKA's continues to surface. Establishing realistic expectations and goals for the planned surgery is imperative and is a responsibility of health-care professionals. If patients are unclear of the purpose and the tangible goals to strive for, the progress of their rehabilitation might be hindered or impeded. This is typically due to the fact that they were not provided or did not establish a clear understanding of what to do at specific times during the recovery/therapy process, nor were they educated on the probable time lines or milestones of their recovery.

Although medicine has continued to progress through the years, patient education has often been excluded in many physician clinics because of operational and fiscal factors. Physicians do not intentionally dismiss or downplay the importance of patient education; however, because of the current state of medicine, operational aspects of their clinic must take precedence. A crucial component that impacts physicians is the time factor. Physicians typically are unable to spend the time with patients to provide an in depth and comprehensive explanation of the patient's entire disease process, all treatment options both conservative and surgical, all risks and benefits, and possible future outcomes.

Although the time surgeons spend with patients in the exam room is limited, the majority of surgeons attempt to provide the purposes and goals of the proposed surgery. However, many factors influence patients before, during, and after their appointments. In one poll, it was reported that approximately 84\% of internet users obtained medical information online and attempted to further their knowledge about specific areas of medicine such as new treatments, medications and surgical procedures [8]. Other influences include media, family, and the patient's peer-based community. All of these factors have a direct influence on the expectations patients formulate before surgery.

\section{Purpose}

The primary purpose of this study was to determine whether the use of a pre-surgical patient education class with an additional long-term expectations module was more effective in modifying participant's pre-surgical expectations than participants receiving the standard pre-surgical educational class alone. Comparing the control group with the intervention group will provide additional data and knowledge to surgeons and educators about the efficacy of addressing patients’ pre-operative expectations and modifying unrealistic expectations into realistic ones. Patients are many times so overwhelmed by their diagnosis, perceived magnitude of the surgical procedure, aggressiveness of the rehabilitation that is required after their surgery, their current pain, and limited functional ability that these individuals do not know what to realistically expect or their expectations may exceed reality. Patients many times lack the knowledge and often fail to inquire about expectations in terms of their specific function and pain after surgery. The research intended to discern construct ways to advocate for patient education courses that would enable patients to generate realistic expectations about their total-knee replacement surgery. In addition, it is anticipated that this research will bring attention to patient-based education 
programs, pre-surgical education, and patient expectations to physicians and health educators in an effort to advocate for further research and implementation of education in the field of medicine.

\section{Methodology}

This study examined whether the use of a pre-surgical patient education class with an additional long-term expectations module which addressed recovery during the first 12 months after surgery, was more effective in modifying participant's pre-surgical expectations than participants receiving the standard pre-surgical educational class alone. Comparing the control group, those who attended the standard pre-surgical patient education class alone, those who attended the standard pre-surgical education class plus the long-term expectations module, provided additional data and knowledge to surgeons and educators about the importance of addressing patient's pre-surgical expectations and modifying unrealistic expectations into realistic ones. The study included one randomized control group to modify pre-surgical expectations of participants undergoing a unilateral total-knee arthroplasty as measured by the Hospital for Special Surgery: Total Knee Replacement Expectation Survey (HSS:TKR Survey).

\subsection{Study Sample}

A total of 42 consecutive unilateral total-knee arthroplasty patients were included in the study. All participants were recruited from two orthopedic clinics in the Central Texas Region. The surgeons participating in the research completed the HSS:TKR Survey for each participant from their own individual perspective as it relates to their diagnosis, co-morbidities, current level of function, and expected future level of function. This was done to establish a score that from the surgeons professional perspective was judged appropriate expectations the participant should have. The surgeon's reported pre-surgical expectations score was then compared to each participants' expectations score. A correlation was then obtained to determine whether there was a difference between the surgeon's pre-surgical expectations and the participants' expectation scores. In addition, a correlation was performed to determine if there were differences between the control and the intervention groups as related to their expectation scores.

\subsubsection{Participants}

Participants included an age range from 50 to 85 and they included a broad range of socioeconomic classes and variations in formal and informal education levels. Participation in the study was voluntary and was limited to patients undergoing their first primary total-joint replacement.

Characteristics for inclusion and characteristics for exclusion in the study are shown in Table 1.

All participants were diagnosed by the treating orthopedic surgeon with osteoarthritis of the knee. This diagnosis was verified and confirmed with radiographic films. Exclusionary criteria for this study included participants having received a previous total-joint replacement such as a shoulder, hip, or knee. Surgical treatment for participants undergoing a revision total-knee arthroplasty due to septic or aseptic loosening of their total-knee components were also excluded. Those with a diagnosis of dementia or a history of other cognitive disorders were disqualified due to their limited cognitive abilities. Individuals who did not retain autonomy of their medical power of attorney were excluded due to the fact they had delegated their medical decisions to a third party. In addition, all patients spoke and read English as their primary language in order to participate in this study.

An established research protocol is shown in Figure 1.

Once the patient decided to schedule their surgery, the patient completed and signed the Participant Consent

Table 1. Characteristics for inclusion and exclusion in the study.

\begin{tabular}{cc}
\hline Characteristics for inclusion & Characteristics for exclusion \\
\hline Volunteered for study & Previous total joint replacement \\
First primary total joint replacement & Diagnosis of dementia \\
Diagnosed with OA of the knee & History of cognitive disorder \\
Diagnosis verified with X-rays & Did not retain medical power of attorney \\
Spoke and read English & \\
\hline
\end{tabular}




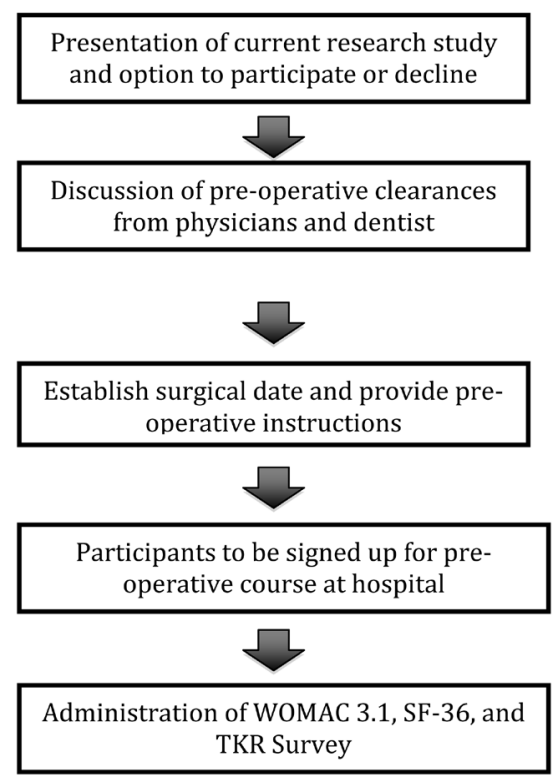

Figure 1. Order of research protocol for data collection.

Form, which was scanned into their electronic medical records. Once the preliminary information was presented and completed, the participant was instructed on the requirements of obtaining clearances from their other medical providers prior to their surgery.

Participants were considered appropriate candidates for a primary unilateral total-knee arthroplasty upon clearance by their primary-care physician, cardiologist, dentist, and orthopaedic surgeon. The pre-surgical education course was presented and the participants determined which date best accommodated their schedule. Each participant was provided a notebook they took to the pre-surgical course and they were encouraged to review the information in the notebook and write down any questions they might have in order to ask the staff teaching the class.

\subsubsection{Instrumentation}

Prior to the participants' surgery and attending the pre-surgical patient education class, three instruments were administered to each participant. The Western Ontario and McMasters University Osteoarthritis Index 3.1 (WOMAC, 3.1), the Medical Outcomes Study Short Form-36 Health Survey (Short-Form-36), and the Hospital for Special Surgery Knee Replacement Expectations Survey (HSS:TKR Survey).

The WOMAC 3.1 Index is a disease-specific, self-administered, health status measurement tool specifically developed for patients with osteoarthritis of the hips and knees [9]. This instrument exhibits excellent reliability and validity when assessing pain, function, and joint stiffness in patients with osteoarthritis of the hips and knees.

The Medical Outcomes Study Short Form-36 Health Survey (SF-36) was designed for the use of clinical practice and research, health-policy evaluations, and general population surveys [10]-[13]. This is a widely accepted general health instrument that is used in various specialties and subspecialties of medicine as a valid and reliable general health survey to measure patients' overall perception of their health.

The Hospital for Special Surgery Total Knee Replacement Expectations Survey (HSS:TKR Survey) addresses symptoms, activities of daily living, walking distance, employment and psychological well-being [14]. This is a valid and reliable instrument to assess pre-surgical patient expectations of knee surgery as well as patient expectations of total-knee replacements.

Variables in this study were comprised of participant gender, age, ethnicity, body-mass index, pre-surgical range of motion, formal education level, WOMAC 3.1 Index, SF-36, pre-education HSS Survey, post-education HSS:TKR Survey, education course with the additional module, education course without the additional module and surgeon's recommended expectations for the HSS:TKR Survey.

A repeated measures ANOVA was used to compare the inter-participant (i.e., within participant) change from 
pre to post-test for both intervention and control groups. The Bonforroni correction was used to control Type I error rate (i.e., to maintain nominal alpha at 0.05 ) for the multiple t-tests. For the between groups comparison (i.e., intervention vs. control), an independent t-test was used for the assessment of change.

\subsubsection{Analysis of the Data}

Forty-two participants were involved in the research study. Nineteen participants attended an intervention class, twenty-three participants attended a control class and all participants completed the follow up HSS: TKA Survey. Both groups were similar with respect to their demographic and clinical characteristics. Individual items addressed in the HSS: TKA Survey were scored from "complete improvement" to "this expectation does not apply to me/I do not have this expectation". The total summed raw score ranged from 0 to 76 and the transformed score (raw score/76 $\times 100$ ) ranged from 0 to 100 . Higher scores indicated the participant expected more improvement for more of the items. The results of the intervention and control groups were similar with respect to the baseline expectation scores.

A paired sample t-test was used to compare the relationship between the surgeon's rated expectation scores to the patient's expectation scores prior to the pre-surgical class.

\section{Results}

The results of the data analysis indicated that for both the intervention and control groups, the surgeons' rated expectations on the HSS:TKR Survey were lower than what the patient had rated for their expectations on both the pre and post-tests on the HSS: TKR Survey. The results were confirmed with the analysis of the t-test which produced the following results, $(\mathrm{t}=5.63, \mathrm{df}=41, \mathrm{p}<0.001)$.

When both the intervention and control groups were compared, results yielded that there was not a statistically significant difference between the pre and post-tests. Neither group demonstrated a significant change between their pre and post-tests with or without the use of the additional long-term expectations module. When the $\mathrm{t}$-test was run, the result produced $(\mathrm{t}=-0.52, \mathrm{df}=41, \mathrm{p}<0.61)$.

When the data were analyzed using a repeated measures ANOVA to compare the group with the module and the group without the module, it was found that there was not a statistically significant difference ( $f(1,40)$ $0.772, \mathrm{p}=0.38$ ). This indicated there was no significant difference in the change of perceptions between the participants in the two treatment groups. In other words, the long-term expectations module did not help the intervention group over the control group as a whole.

When the level of education was used as a variable, a simple linear regression analysis was performed on the intervention group. The results approached a statistically significant difference when the long-term expectations module was used $\left(\mathrm{t}=-1.78, \mathrm{p}=0.09, \mathrm{R}^{2}=0.16\right)$. This compares to the control group where there was no significant change $\left(t=-0.78, p=0.44, R^{2}=0.03\right)$. The $R^{2}$ is the coefficient of the determination and is a measure of practical significance. The $\mathrm{R}^{2}$ of 0.16 would suggest that a larger sample would have found a statistical significance. Therefore, we can conclude that the long-term expectations module was potentially more effective with participants having higher than a high-school education.

A new variable was created by splitting the participants into two groups, those with a high-school education or less and those with more than a high-school education. Indeed, the module had more effect on those participants with higher than a high-school education $(\mathrm{t}=2.31 \mathrm{df}=17 \mathrm{p}=0.03)$. For those participants that did not receive the module, there was no difference based on their education $(t=0.87, \mathrm{df}=21, \mathrm{p}=0.39$ ).

\section{Discussion}

Patients preparing to have a total-knee replacement have multiple concerns and expectations related to their long-term function and outcomes. In general, patient expectations after a total-joint replacement can be quantified simply as relief of pain and being able to function freely [15]. This study set out to determine if the use of an additional long-term expectations module would help improve expectations that patients had already formed as they relate to long-term recovery.

Medical professionals must be aware of the patient's need for pre-surgical education due to the evolving nature of medicine. As the industry evolves, patients are now demanding more from their care givers. With the consumer-driven nature of the health care industry, it is becoming more essential than ever that hospitals and other health care providers develop reliable approaches that facilitate and promote patient autonomy. In addition, 
as the need for medical care increases and the number of surgeons decreases, the time surgeons and their staff are able to spend with patients in a one-on-one setting is limited. Therefore the need for alternative methods of addressing these problems must be addressed. Although pre-surgical education and patient autonomy is essential, we must also take into consideration the ability for patients to access information via the internet. Providing reliable, non-proprietary, quality education to patients will perhaps provide a safe haven where patients can feel confident that they can refer to a source to have their concerns addressed without contradictions in the information they are obtaining. Patients need education not just about the pre-surgical aspects of their care, but they need to be educated and made aware of the milestones that should be reached during various times of their recovery.

Although, as a whole there was not a statistically significant difference between the two groups' expectations scores, it was remarkable that when the level of education was factored into the equation, participants receiving the additional module and who had higher than a high-school education, had post-surgical expectations that were more aligned with the surgeons' expectations. Interpretation of these results can assist in targeting the underserved and less educated population. These results indicate that health care professionals must take into account various adult learning methodologies [16]-[19] and other adult-learning theorists as pre-surgical education relates to those with less formal education. Being able to provide appropriate education and information to patients is essential due to the fact that when educating patients, health care providers are wanting and expecting the individuals to implement the learned information into their lives since it will have an impact on their physical health.

Creating comprehensive and appropriate patient education programs will help decrease the burden on the physicians while increasing the patients' autonomy in their care. Not only does patient education allow for patient autonomy, it also assists their social and familial support systems by means of allowing these family to know and understand the purpose of the surgery and the best way to facilitate assistance in having the patient resume their functional activities and lives. In return, the overall burden on the family and other non-professional providers in the patient's post-surgical care would decrease because they are also being educated on recovery and other post-surgical milestones. In addition, it can be argued that by educating patients pre-surgically about surgical milestones such as range of motion for knees, precautions, and care of wounds, this would reduce the overall number of re-admittance visits to the hospital because patients would be able to identify problems early and not allow the problems to escalate to a critical point where hospitalization was required.

\section{Conclusion}

In conclusion, as medicine moves towards being more consumers driven, hospitals and other medical facilities should begin exploring various methods of providing patient education. Creating and implementing quality patient-education programs can be enormously beneficial not only to the patients, but also to the health care industry. Health care providers must not only be aware of the accuracy of the content that is being put together, but also be very aware of the use and implementation of adult-learning strategies, methodology, and health literacy. Healthcare is on the verge of significant change; therefore, health care providers and educators are wise to begin looking at patient education through a different lens.

\section{References}

[1] Kurtz, M.S., Ong, K., Lau, E., Mowat, F. and Halpern, M. (2007) Projections of Primary and Revision Hip and Knee Arthroplasty in the United States from 2005-2030. The Journal of Bone and Joint Surgery, 89, 780-785. http://dx.doi.org/10.2106/JBJS.F.00222

[2] Lingard, E., Sledge, C. and Learmonth, I. (2006) Patient Expectations Regarding Total Knee Arthroplasty: Differences among the United States, United Kingdom, and Australia. The Journal of Bone and Joint Surgery, 88, 1201-1207. http://dx.doi.org/10.2106/JBJS.E.00147

[3] Jeffries, M.A., Donica, M., Baker, L.W., Stevenson, M.E., Annan, A.C. and Humphrey, M.B. (2014) Genome-Wide DNA Methylation Study Identifies Significant Changes in Osteoarthritic Cartilage. Arthritis Rheumatology, 66, 28042815. http://dx.doi.org/10.1002/art.38762

[4] Felson, D.T. (2006) Clinical Practice. Osteoarthritis of the Knee. New England Journal of Medicine, 354, 841-848. http://dx.doi.org/10.1056/NEJMcp051726

[5] Sharma, A.R., Jagga, S., Lee, S. and Nam, J. (2013) Interplay between Cartilage and Subchondral Bone Contributing to 
Pathogenesis of Osteoarthritis. International Journal of Molecular Sciences, 14, 19805-19830. http://dx.doi.org/10.3390/ijms141019805

[6] Dahlen, L., Zimmerman, L. and Barron, C. (2006) Pain Perception and Its Relationship to Functional Status Post Total Knee Arthroplasty: A Pilot Study. Orthopaedic Nursing, 25, 264-270. http://dx.doi.org/10.1097/00006416-200607000-00009

[7] Soohoo, N., Sharifi, H., Kominski, G. and Lieberman, J. (2006) Cost Effectiveness Analysis of Unicompartmental Knee Arthroplasty as an Alternative to Total Knee Arthroplasty for Unicompartmental Osteoarthritis. The Journal of Bone and Joint Surgery, 88, 1975-1982. http://dx.doi.org/10.2106/JBJS.E.00597

[8] Ullrich, P.F. and Vaccaro, A.R. (2002) Patient Education on the Internet: Opportunities and Pitfalls. Spine, 27, 185188. http://dx.doi.org/10.1097/00007632-200204010-00019

[9] Bellamy, N. (2008) Western Ontario and McMasters University Index 3.1 User’s Guide IX.

[10] McHorney, C.A., Ware, J.E. and Raczek, A.E. (1993) The MOS 36-Item Short Form Health Survey (SF-36) II Psychometric and Clinical Tests of Validity in Measuring Physical and Mental Constructs. Medical Care, 31, 247-263. http://dx.doi.org/10.1097/00005650-199303000-00006

[11] Stansfeld, S.A., Roberts, R. and Foot, S.P. (1997) Assessing the Validity of the SF-36 General Health Survey. Quality of Life Research, 6, 217-224. http://dx.doi.org/10.1023/A:1026406620756

[12] Tellini, A., Ciccone, V., Blonna, D., Rossi, R, Marmotti, A. and Castoldi, F. (2007) Quality of Life Evaluation in Patients Affected by Osteoarthritis Secondary to Congenital Hip Dysplasia after Total Hip Replacement. Journal of Orthpaedic Traumatology, 9, 155-158. http://dx.doi.org/10.1007/s10195-008-0022-6

[13] Ware, J.E. and Sherbourne, C.D. (1992) The MOS 36-Item Short-Form Health Survey (SF-36). Medical Care, 30, 473483. http://dx.doi.org/10.1097/00005650-199206000-00002

[14] Mancuso, C.A., Sculco, T., Wickiewics, T., Jones, E., Robbins, L., Warren, R. and Williams-Russo, P. (2001) Patients' Expectations of Knee Surgery. Journal of Bone and Joint Surgery, 83, 1005-1012.

[15] Suda, A., Seeger, J., Bitsch, R., Clarus, M. and Drueger, M. (2010) Are Patient Expectations of Hip and Knee Arthroplasties Fulfilled? Orthopedics, 33, 76-80. http://dx.doi.org/10.3928/01477447-20100104-07

[16] Knowles, M.S., Holton, E.F. and Swanson, R.A. (2005) The Adult Learner: The Definitive Classic in Adult Education and Human Resource Development. Elsevier, Burlington.

[17] Kolb, D.A. (1984) Experimental Learning: Experience as the Source of Learning and Development. Prentice-Hall, Englewood Cliffs.

[18] Kolb, D.A., Boyatzis, R.E. and Mainemilis, C. (2000) Experimental Learning Theory: Previous Research and New Directions. In: Sternberg, R.J. and Zhang, L.F., Eds., Perspectives on Cognitive Learning, and Thinking Styles, New Jersey.

[19] Dewey, J. (1938) Experience and Education. Kappa Delta Pi, West Lafayette.

\section{Abbreviations}

OA: Osteoarthritis

TKA: Total Knee Arthroplasty

HSS: TKR Survey: Hospital for Special Surgery: Total Knee Replacement Expectations Survey

WOMAC 3.1: Western Ontario Macmasters University Osteoarthritis Index 3.1

SF-36: Medical Outcomes Study Short Form-36 